\title{
VIDA ODONTO: Ambiente de Realidade Virtual para Treinamento Odontológico
}

\author{
Title: VIDA ODONTO: Virtual Reality Environment for Dental Training
}

\author{
Romero Tori \\ Escola Politécnica \\ Universidade de São Paulo \\ tori@usp.br \\ Allan Amaral Tori \\ VRMonkey \\ Univesp \\ allant22@gmail.com
}

\author{
Gustavo Ziyu Wang \\ Escola Politécnica \\ Universidade de São Paulo \\ fgustavo.ziyu@gmail.com \\ Elen Collaço de Oliveira \\ Escola Politécnica \\ Universidade de São Paulo \\ elencollaco@gmail.com
}

\author{
Lucas Henna Sallaberry \\ Escola Politécnica \\ Universidade de São Paulo \\ lhsallaberry@gmail.com \\ Maria A.A.M. Machado \\ Fac. Odontologia de Bauru \\ Universidade de São Paulo \\ mmachado@fob.usp.br
}

\section{Resumo}

O treinamento de procedimentos médicos invasivos na etapa pré-clinica é um grande desafio educacional. A abordagem tradicional, com animais e cadáveres, apresenta problemas éticos e práticos. Uma solução promissora é a utilização de realidade virtual $(R V)$, que permite simulações realistas de baixo custo, repetibilidade e feedback em tempo real. Tal constatação motivou o desenvolvimento do ambiente VIDA Odonto, o qual utiliza tecnologias avançadas de RV, modelagem realista do paciente e interação imersiva tridimensional. O protótipo desenvolvido, do módulo do ambiente virtual desse sistema, foi testado e avaliado por profissionais com experiência na prática clínica e principiantes, demonstrando a viabilidade de se tornar um recurso educacional relevante em cursos de odontologia.

Palavras-chave: realidade virtual, odontologia, treinamento, imersão, interação 3D.

\begin{abstract}
Training invasive medical procedures at the pre-clinical development is a considerable educational challenge. The traditional approach, with animals and corpses, presents both ethical and practical issues. So, the use of virtual reality $(V R)$, which enables low-cost realistic simulations, repeatability and real time feedback, is a promising solution. Such ascertainment motivated the development of the virtual environment VIDA Odonto, which resorts to VR technologies, realistic modeling of patient and tridimensional immersive interaction. The developed prototype of the virtual environment of such system was tested and evaluated by professionals with clinical experience and beginners, demonstrating its viability to be a relevant educational resource in dentistry courses.
\end{abstract}

Keywords: virtual reality, odontology, training, immersion, 3D interaction.

Cite as: Tori, R., Wang, G.Z., Sallaberry, L.H., Tori, A.A., Oliveira, E.C., E Machado, M.A.A.M. (2018). VIDA ODONTO: Virtual Reality Environment for Dental Training (VIDA ODONTO: Ambiente de Realidade Virtual para Treinamento Odontológico). Brazilian Journal of Computers in Education (Revista Brasileira de Informática na Educação - RBIE), 26(2), 80-101. DOI: 10.5753/RBIE.2018.26.02.80. 


\section{Introdução}

Treinar futuros médicos, enfermeiros ou dentistas traz grandes desafios por envolver seres humanos, pela necessidade de infraestrutura de alto custo e pela complexidade e delicadeza dos procedimentos. Para o ensino de anatomia, a base de qualquer formação na área de saúde que envolve a capacitação de profissionais que atenderão pacientes, costumam ser utilizados diversos meios, tais como laboratórios que possibilitam a manipulação de corpos humanos e de animais, modelos e peças anatômicas, vídeos, imagens e multimídia (Battulga, Konishi, Tamura, \& Moriguchi, 2012). Existem diversos problemas nessa abordagem tradicional. A manipulação de organismos apresenta questões éticas, morais e culturais. Além disso, restos de corpos humanos não fornecem respostas fisiológicas aos estudantes que os manipulam, diminuindo a percepção de realismo (McLachlan, Bligh, Bradley, \& Searle, 2004). A maneira como a morte é apresentada aos alunos é um problema adicional. Estudantes expostos a cadáveres apresentam uma maior frequência de alterações psicológicas e mentais, de acordo com estudos realizados nos Estados Unidos e Austrália (Gustavson, 1988) (Hancock, Williams, \& Taylor, 1998). Eles podem inclusive desenvolver atitudes inapropriadas para lidarem com o estresse causado por essas aulas (Finkelstein \& Mathers, 1990). Apesar desses problemas, o aprendizado baseado nas mídias atuais, como livros e tutorias virtuais, ainda não obteve êxito em substituir a dissecação. Uma das principais críticas dos estudantes de anatomia, nesse caso, é a falta de percepção de profundidade (Azer \& Eizenberg, 2007). Treinamentos auxiliados por dispositivos de realidade virtual e reconhecimento de gestos, por outro lado, possibilitam realismo, percepção de imersão (Vora et al., 2002) (Xu, 2006), por meio da visão estereoscópica, e repetibilidade dos procedimentos, com baixo custo e sem os problemas éticos e logísticos presentes em formas tradicionais de treinamento.

A partir dos resultados obtidos no projeto VIDA (Virtual Interactive Distance-learning on Anatomy) (Melo, Aceiro, Nunes, Tori, \& Muntoreanu, 2011) (R. Tori, Nunes, Gomes, \& Tokunaga, 2009) (R. Tori, Marques, et al., 2009), em que foram desenvolvidas interfaces interativas tridimensionais imersivas, e da experiência adquirida com o projeto Vimet Odonto (Correa, 2015), que gerou uma interface háptica para simulação de anestesia do nervo alveolar inferior, foi desenvolvido e avaliado o protótipo do módulo de ambiente virtual que comporá uma ferramenta imersiva, baseada em interação tridimensional, para treinamento de anestesia odontológica, denominada VIDA Odonto. Essa ferramenta de baixo-custo, quando comparada aos instrumentos educacionais convencionais citados, poderá ser utilizada presencialmente ou a distância, com capacete HMD (Head-Mounted Display) ou com adaptadores de baixo custo para smartphones. A ferramenta também avalia automaticamente a precisão do procedimento realizado pelo treinando e permite a gravação para futura avaliação pelo professor ou pelo próprio aluno. Tal recurso ainda pode ser usado na preparação de vídeos didáticos imersivos. Enquanto o foco do trabalho de Corrêa (Correa, 2015) foi o realismo da interface háptica, o trabalho aqui apresentado tem como objeto de estudo a imersão e o realismo do ambiente virtual onde o treinamento será realizado.

Este artigo é uma versão estendida e atualizada do trabalho apresentado no $\mathrm{V}$ Congresso de Informática na Educação (R. Tori et al., 2016), tendo sido premiado como o 
melhor artigo da trilha 2 do SBIE (Simpósio Brasileiro de Informática na Educação, evento integrante do CBIE): "Jogos, simulação, gamificação, meta-cognição e neurociência em Ambientes e Sistemas Computacionais para Ensino/Aprendizagem".

O presente artigo está organizado em seções. A seção 2 descreve os cenários e objetivos desse trabalho. As interações em ambientes tridimensionais são descritas na seção 3. Outros sistemas, relacionados ao projeto aqui apresentado, são descritos na seção 4. A arquitetura concebida para o ambiente VIDA Odonto é descrita na seção 5. Ainda na seção 5, são descritos o método de avaliação automática, a modelagem 3D e a prova de conceito. O planejamento e a preparação do experimento piloto são descritos na seção 6. Os resultados do experimento são relatados e analisados na seção 7. Por fim, na seção 8, são apresentadas as conclusões e as investigações futuras.

\section{Cenários e objetivos}

Os estudantes de odontologia precisam passar por treinamentos pré-clínicos sucessivos antes de executarem pela primeira vez o procedimento de anestesia em uma pessoa real. A exemplo de pilotos de avião, e de outras profissões que envolvem a integridade e saúde de seres humanos, não é tolerável que uma pessoa não adequadamente treinada, avaliada e aprovada seja liberada para atuação profissional. No caso dos pilotos já é imperativo o uso de simuladores de vôo. Em um futuro próximo é provável que estudantes da área de saúde também tenham o treinamento em simuladores incluídos em suas grades curriculares.

Em parceria com a Faculdade de Odontologia de Bauru (FOB/USP), está sendo desenvolvido pelos autores, com a participação de outros pesquisadores, alunos e colaboradores, um ambiente imersivo para treinamento de procedimentos odontológicos, que foi denominado "Laboratório de Simulação e Treinamento"(LaSIT), do qual a ferramenta VIDA Odonto fará parte. O primeiro procedimento a ser testado com essa ferramenta é o de anestesia do nervo alveolar inferior. Posteriormente o ambiente VIDA Odonto será expandido a fim de possibilitar treinamentos de outros procedimentos da rotina da clínica odontológica, que poderão englobar desde a obtenção e análise de imagens de radiografias até a esterilização e organização de instrumentais, passando por interações com o paciente e suas reações emocionais e físicas. Por esse motivo está sendo modelado um consultório dentário (Figura 1a) e um paciente de 10 anos de idade, em corpo inteiro e com alto detalhamento interno das estruturas anatômicas da boca, incluindo maxilar e mandíbula (Figura 1b) (A. Tori, Leite, Pacolla, Amantini, \& Machado, 2014).

A ferramenta está sendo projetada de forma a prover ao estudante de odontologia nas etapas iniciais de sua formação pré-clínica, sensações de presença (Biocca, Harms, \& Gregg, 2001) (R. Tori, 2010) e imersão, isto é, provocar no usuário a sensação de estar envolvido com o ambiente odontológico e com pacientes virtuais e poder interagir como se estivesse em situações próximas àquelas que ocorrem no aprendizado em um laboratório real. Laboratórios virtuais imersivos de alta tecnologia, baseados em CAVE, capacetes HMD profissionais, luvas de dados e outros dispositivos acoplados ao corpo 

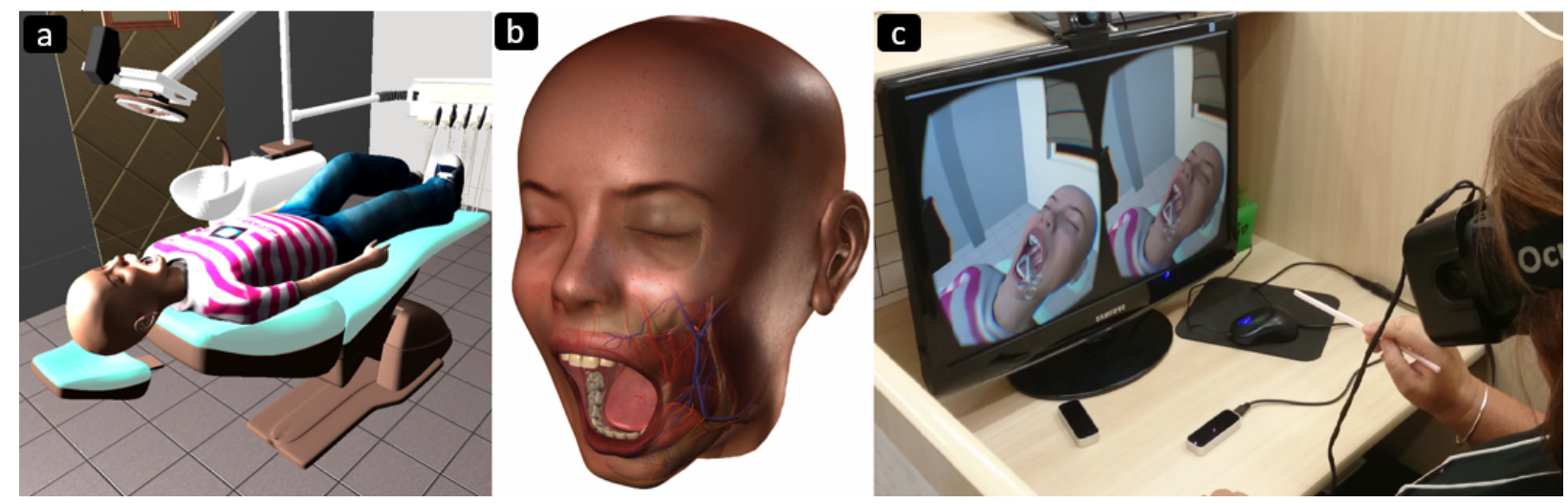

Figura 1: Consultório odontológico virtual, modelo 3D da cabeça e simulação da aplicação de uma injeção no VIDA Odonto.

(R. Tori, Marques, et al., 2009), atenderiam tais requisitos, mas por apresentarem altos custos (alguns não são produzidos no Brasil exigindo importação e são pouco flexíveis), inviabilizariam o uso em alta escala.

A hipótese inicial do VIDA Odonto é que, com técnicas de reconhecimento de gestos e de imersão em ambiente tridimensional, é viável a criação de um ambiente de baixo custo que possibilite ao estudante de odontologia treinar, numa das etapas de formação pré e pós clínica, procedimentos de anestesia de forma intuitiva, natural, com baixa sobrecarga cognitiva e alta percepção de presença, como se estivesse realizando-os em pacientes reais.

O experimento apresentado neste artigo teve por objetivo desenvolver e testar um protótipo imersivo de um consultório odontológico dotado de funcionalidades básicas para a realização de um procedimento clínico fundamental para a prática clínica, que é a anestesia. Esse ambiente virtual comporá o módulo central do sistema VIDA Odonto, ao qual serão acoplados novos módulos, como o de interface háptica e o de participação do avatar do professor a distância, entre outros. O protótipo aqui apresentado permite ao operador realizar o movimento inicial da seringa, avaliação de sua precisão e gravação do procedimento. $\mathrm{O}$ teste da primeira etapa do movimento (aproximação da seringa e posicionamento sobre o ponto de inserção) foi realizado por profissionais e professores experientes, bem como por alunos iniciantes de odontologia e de outros cursos (simulando alunos sem qualquer experiência). Os movimentos foram gravados e avaliados, quanto a sua precisão, pelo módulo de avaliação automatizada. Foi também avaliado o grau de realismo no espaço virtual percebido pelos participantes durante a realização do procedimento.

\section{Interações em Ambientes Tridimensionais}

A interação em ambientes virtuais tridimensionais (AV3D) se dá por meio de operações de manipulação de objetos com vistas a atingir objetivos específicos. Para cada operação realizada o AV3D deve produzir um feedback adequado. Segundo Pinho e Rebelo (Rebelo 
\& Pinho, 2004), são três os elementos de uma interface homem-máquina: dispositivo de entrada, que captura ações ou estímulos gerados pelos usuários; função de transferência, que mapeia a ação capturada para um elemento ou elementos controlados pelo sistema; e dispositivo de saída, que envia uma resposta à ação executada.

As interações devem ser projetadas levando em consideração características e limitações dos dispositivos de interface, para que sejam adequadas ao cenário e ao público-alvo a que se destinam. Segundo Bowman e seus colegas (Bowman, Kruijff, LaViola Jr, \& Poupyrev, 2001), as operações podem ser agrupadas em três categorias: navegação, seleção/manipulação e controle do sistema. A navegação consiste no movimento do usuário dentro do AV3D, a seleção/manipulação se refere à escolha de um objeto virtual e à modificação de suas características, e o controle do sistema define comandos específicos para modificar o estado do sistema.

Em AV3D voltados para a educação médica, um cuidado especial deve ser tomado com a precisão na modelagem do ambiente, equipamentos e, principalmente, das partes orgânicas que serão manipuladas pelo sistema. Por se destinar a uso em educação a distância, deve também ser levada em conta a diversidade de equipamentos, plataformas e configurações que os usuários remotos terão. Para atender às necessidades expostas foram incluídos os seguintes requisitos: a disponibilidade para uso de dispositivos de entrada e saída de baixo custo (requisito de sistema) e a naturalidade do processo de interação (requisito de interface).

\section{Sistemas Relacionados}

São discutidos a seguir sistemas que de alguma forma se relacionam com o VIDA Odonto, seja pela influência direta que exerceram no projeto, seja por algum nível de similaridade (objetivos, tecnologia ou abordagem). São eles: VIDA (originalmente destinado para o ensino de anatomia e que evoluiu para o VIDA Odonto); Vimet (solução anterior para o mesmo problema, cujos requisitos e resultados inspiraram a concepção do VIDA Odonto); Haptic Dental Injection Simulation (sistema com objetivos similares, porém com tecnologia, paradigma de interface e abrangência diferentes ); Dental Anesthesia Simulator (sistema com objetivos similares, porém com tecnologia, paradigma de interface e abrangência diferentes); Simodont (Sistema comercial para treinamento odontológico, mais abrangente e de alto custo, porém menos imersivo que o VIDA Odonto); Outros simuladores (síntese dos principais estudos envolvendo simuladores no treinamento odontológico).

\subsection{VIDA}

O projeto VIDA (Virtual Interactive Distance-learning on Anatomy) (Melo et al., 2011) (R. Tori, Marques, et al., 2009) (R. Tori, Nunes, et al., 2009), propôs um novo paradigma de interação, denominado holográfico, cujo princípio é o de dar ao usuário a sensação de estar manipulando os objetos anatômicos virtuais com as mãos livres, como se fossem 
holografias e possuíssem volume.

Por ser destinado a cursos a distância e ter como requisito o baixo custo, a melhor solução na época foram os óculos de anaglifo. Toda a interação do VIDA é baseada em gestos naturais, que foram definidos a partir de estudos etnográficos realizados com potenciais usuários. Para evitar menus, que quebrariam a ilusão do efeito holográfico, comandos de sistema são acionados por voz. Os gestos deveriam ser necessariamente naturais, como se os objetos fossem manipulados fisicamente, nunca gestos de comando. Os princípios de interface do ambiente VIDA foram transferidos para o VIDA Odonto, com a diferença que este passa a usar tecnologia de software e equipamentos de imersão de realidade virtual mais atuais, que melhor atendem hoje aos requisitos do sistema.

\subsection{Vimet Odonto}

O sistema Vimet Odonto (Correa, 2015) teve como objetivo a simulação da anestesia do nervo alveolar inferior para treinamento odontológico. Diferentemente do ambiente objeto deste trabalho, cujo foco principal é a interação imersiva, o foco do Vimet Odonto foi a interação háptica. Por utilizar Java e Java 3D não foi possível aproveitar o que já foi desenvolvido no Vimet para incorporação no VIDA Odonto. Pretende-se, no entanto, utilizar todo o conhecimento gerado pelo projeto Vimet Odonto para incorporação de um futuro módulo de interface háptica no sistema.

\subsection{Haptic Dental Injection Simulation}

Um simulador de realidade virtual para treinamento da aplicação de anestesia para bloqueio do nervo alveolar inferior foi desenvolvido e utilizado em faculdades de odontologia na Escócia para familiarizar alunos com o procedimento (Poyade, Lysakowski, \& Anderson, 2014). O simulador conta com um modelo anatomicamente preciso de uma cabeça e pescoço humanos, incluindo os músculos, nervos, ossos e vasos sanguíneos. Utiliza-se uma interface háptica para o retorno de força da seringa penetrando os diferentes tecidos.

Outros pontos levantados nesse trabalho foram a limitação do alcance do aparelho háptico e utilização da ferramenta padrão desse aparelho, que foge muito ao usado pelos dentistas, a seringa, para realizar o treinamento. Essas limitações podem ser amenizadas pela utilização de uma tecnologia de rastreamento de objetos, como a utilizada no VIDA Odonto, permitindo um espaço de trabalho mais amplo, além de aceitar diferentes instrumentos para rastreamento.

\subsection{Dental Anesthesia Simulator}

Ainda no contexto de anestesia do nervo alveolar inferior, um simulador desenvolvido na Unicamp (Pereira, 2016) utiliza técnica de realidade aumentada e uma seringa real como formas de interação física e, portanto, o usuário interage com dois marcadores de papel (cabeça e seringa).

O simulador conta com um modelo com características anatômicas - tecidos moles e 
ossos - de uma boca natural. Contudo essa solução se limita ao realismo visual das estruturas anatômicas da região da boca, havendo lacunas no grau de realismo do detalhamento interno das estruturas anatômicas incluindo maxilar e mandíbula.

\subsection{Simodont}

O simulador comercial para treinamento odontológico desenvolvido pela Moog Inc. (Bakr, Massey, \& Alexander, 2013) utiliza tecnologia de realidade virtual, porém não imersiva, e é utilizado para auxiliar no aprendizado em algumas universidades. Esse simulador apresenta monitor 3D e feedback háptico, além de permitir diversos procedimentos odontológicos. Uma avaliação automática é também utilizada para determinar o desempenho do aluno, assim como um replay em vídeo que permite o professor analisar as técnicas do aluno. O VIDA Odonto, em contrapartida, visa ser uma ferramenta de baixo custo e de código aberto, facilitando não apenas sua adoção como recurso didático como também a realização de pesquisas científicas.

\subsection{Outros Simuladores para treinamento odontológico}

Um estudo foi realizado na West China Dental School (Ren et al., 2017) com o objetivo de analisar a resposta de alunos a simuladores digitais. Quatro diferentes tecnologias foram apresentadas aos estudantes, sendo elas microscópios digitais, slides virtuais de patologia, imagens digitais de raio $X$ e simuladores virtuais. Os resultados apresentados mostraram alto grau de aceitação para todas as tecnologias, com $81,2 \%$ dos estudantes apontando os simuladores virtuais como uma experiência positiva. Além disso, o simulador foi também apontado como a tecnologia favorita entre as quatro por $54,6 \%$ dos alunos, tendo o aumento do entusiasmo e motivação, além de respeitar a velocidade de aprendizado do aluno como alguns dos pontos positivos. O custo do aparelho, porém, se mostrou uma preocupação para $48.9 \%$ dos alunos. A capacidade dos simuladores de gravar vídeos com o desempenho do aluno foi apontado pelos autores como uma experiência reveladora para o estudante, encorajando sua auto reflexão e auto avaliação.

Outro estudo com alunos da School of Dentistry at the University of Leeds (Mirghani et al., 2016) foi realizado entre estudantes do primeiro, terceiro, quarto e quinto anos de graduação, utilizando-se o Simodont para avaliar a capacidade do simulador de diferenciar estudantes com variados níveis de experiência no mundo real. Utilizando dados fornecidos pelo sistema, o desempenho dos alunos foi analisado enquanto realizavam um procedimento simples no simulador. Os resultados obtidos mostraram que os dados fornecidos eram capazes de diferenciar alunos iniciantes (primeiro ano) de experientes (terceiro, quarto e quinto anos) e foi sugerido que procedimentos mais complexos devem ser capazes de diferenciar alunos de terceiro, quarto e quinto anos quanto ao seu grau de experiência, mostrando a viabilidade de se utilizar avaliações automáticas do sistema. 


\section{VIDA Odonto}

O sistema VIDA Odonto (R. Tori et al., 2016) utiliza realidade virtual imersiva para oferecer um ambiente virtual para treinamento de procedimentos odontológicos, aplicando os conceitos de manipulação direta e interface holográfica do ambiente VIDA.

Sendo o sistema um ambiente de aprendizagem que terá constante expansão, nesta seçãoé apresentada a arquitetura projetada para o sistema expandido. Seu desenvolvimento é interdisciplinar, acompanhado por especialistas da área de odontologia, design e engenharia.

O protótipo implementado do módulo de ambiente virtual, em relação a outros sistemas existentes, apresenta os seguintes diferenciais:

- interface holográfica de manipulação direta (entenda-se aqui "paradigma holográfico"como sendo a simulação de se interagir com a projeção de objetos virtuais volumosos, como se esses estivessem ao alcance das mãos do interator - usuário interagindo com o sistema -, sem intermediação, seja por apontadores ou elementos de interface);

- imersão: possibilidade de se movimentar pelo consultório virtual e se posicionar como se estivesse ao lado da cadeira do paciente;

- avaliação automática da precisão do movimento;

- gravação do experimento para posterior visualização imersiva.

\subsection{Arquitetura do Sistema}

A arquitetura planejada para o ambiente VIDA Odonto é apresentada nas Figuras 2 e 3. Ainda que o objetivo da pesquisa aqui relatada seja focado apenas no ambiente virtual, é importante que esse esteja contextualizado em um objetivo mais amplo, no qual se insere. O ambiente virtual é a essência do sistema VIDA Odonto. Por esse motivo foi o primeiro módulo a ser desenvolvido e validado, cujos resultados são apresentados neste artigo.

Os demais módulos encontram-se em desenvolvimento, sendo objetos de novas pesquisas, as quais foram viabilizadas pelos resultados do desenvolvimento do ambiente virtual. Durante todo o desenvolvimento desse ambiente a arquitetura foi levada em consideração no estabelecimento de seus requisitos e nas decisões de projeto, de forma que já estivesse preparado para as expansões futuras.

A seguir os principais módulos são discutidos. 


\section{Diagrama Físico}



Figura 2: Arquitetura concebida para o ambiente VIDA Odonto (visão geral das partes físicas do sistema).. 


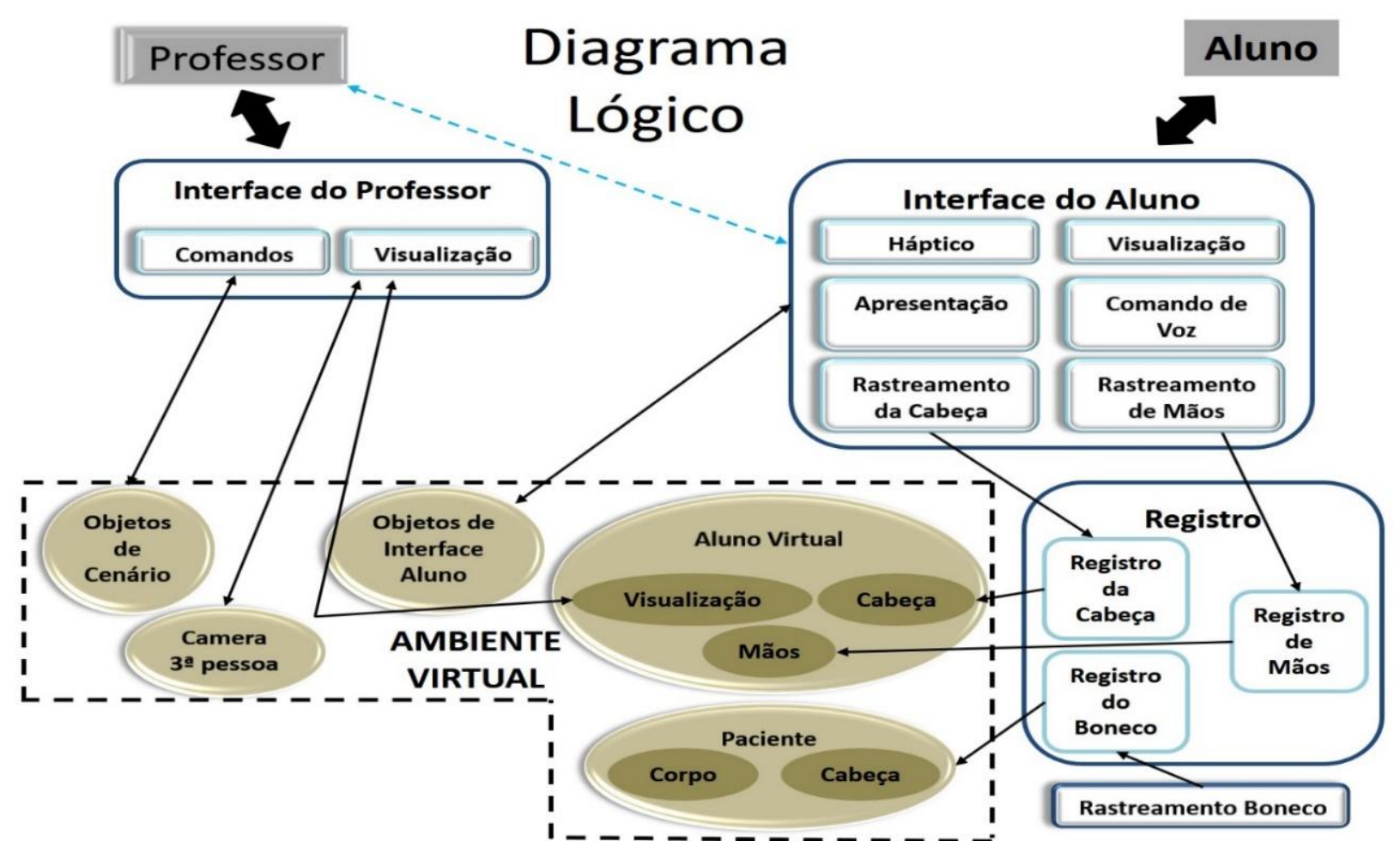

Figura 3: Arquitetura concebida para o ambiente VIDA Odonto (visão geral das partes lógicas do sistema)..

\section{Ambiente Virtual}

O ambiente virtual é composto por consultório, aluno, paciente, objetos de cenário, objetos de interface e câmera. Futuramente haverá também o avatar do professor, que poderá interagir a distância. Esse é o módulo que foi desenvolvido e avaliado na pesquisa aqui relatada. Houve uma preocupação com o registro entre o posicionamento físico do aluno em relação ao consultório virtual, de forma que ele faça os movimentos corporais e se sinta como se estivesse em um consultório real. Todas as proporções dos elementos de cena e do paciente foram estabelecidas com base nesse princípio. Foi também previsto o rastreamento da cabeça e das mãos do aluno, para garantir o registro e o correto posicionamento da câmera virtual. A Figura 4 mostra como é a imersão do aluno nesse ambiente. 


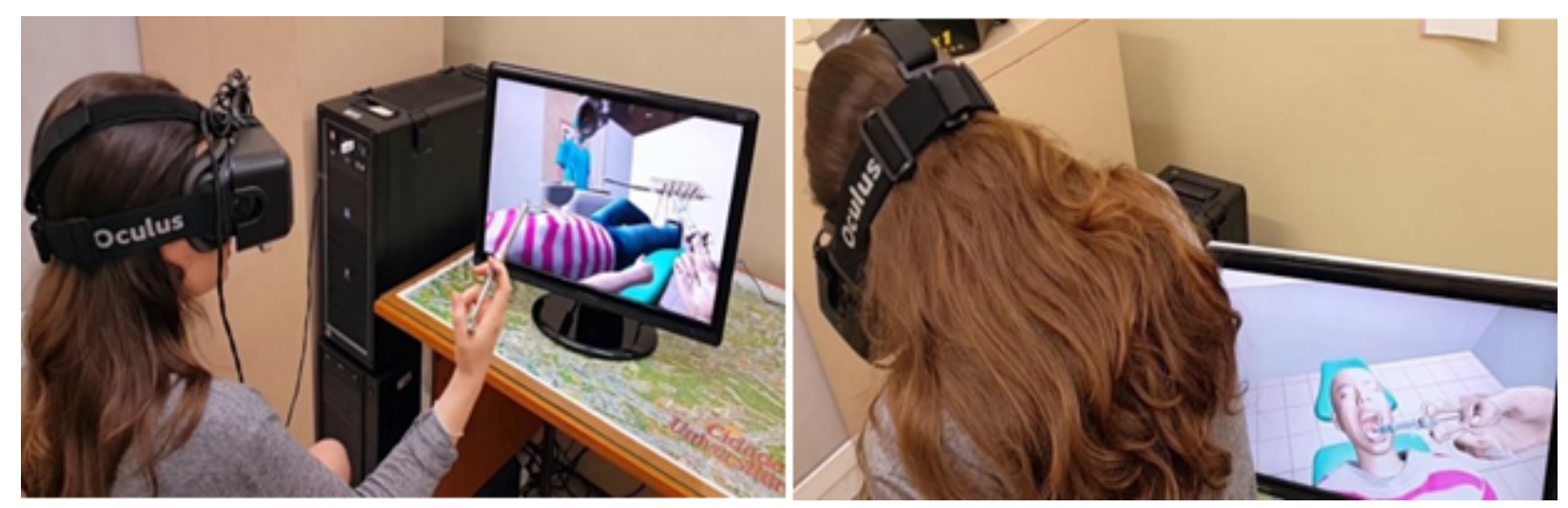

Figura 4: Imersão no ambiente virtual do VIDA Odonto.

Por meio de um sensor de profundidade (Leap Motion) especializado na detecção de movimentos de mãos, acoplado ao capacete de realidade virtual (HMD), no caso o Oculus Rift DK2, que possui rastreamento da posição da cabeça, o aluno visualiza suas mãos dentro do consultório virtual e pode se movimentar como se estivesse no consultório real. Para aumentar o realismo o aluno segura uma seringa carpule verdadeira (instrumento odontológico utilizado para aplicação de anestesia) e visualiza suas mãos e seringa virtual exatamente nas mesmas posições em que se encontram no mundo real.

\section{Interface do aluno}

A interface do aluno, que não fez parte da pesquisa aqui apresentada, será composta por um sistema háptico (para retorno de força durante o procedimento de aplicação da anestesia, e também por um boneco que poderá ser tocado e sua boca manipulada, com reflexo imediato na boca do paciente virtual), rastreamento de mãos e cabeça (já implementado), comando de voz (para que o aluno não precise utilizar mouse ou teclado nem interagir com menus flutuantes no ambiente virtual, que reduziriam a sensação de presença e realismo).

\section{Interface do professor}

O professor poderá interagir a distância, acompanhando tudo o que o aluno fizer em tempo real ou assistindo a gravações dos treinamentos. Essas gravações não são vídeos comuns, mas sim reproduções imersivas em $3 \mathrm{D}$, sob o ponto de vista do aluno. $\mathrm{O}$ professor poderá assistir a essas gravações na tela do computador ou usando um capacete de realidade virtual. O professor também poderá interferir no ambiente, modificando os estados e instrumentos, mobiliário e paciente. Também haverá a possibilidade de trocar de papel com o estudante, tanto para fazer o procedimento enquanto o aluno assiste pelos olhos do professor, como assisti-lo, pelos olhos do aluno, quando esse é realizado. Por fim será possível avaliar o procedimento, tendo ou não como referência a avaliação automática gerada pelo sistema.

\section{Registro}

Para que a imersão e a experiência do usuário sejam as mais realistas possíveis, é essencial que os elementos físicos (cabeça, mãos, seringa e boneco) permaneçam 
perfeitamente registrados no espaço com seus correspondentes virtuais. Este módulo se encarregará de calibrar, monitorar e ajustar automaticamente todos os posicionamentos e dimensionamentos do sistema.

\subsection{Avaliação Automática}

Um dos recursos inovadores do VIDA Odonto é a possibilidade de avaliação automática do desempenho do treinando durante a realização do procedimento. Para tanto o sistema prevê a gravação de todo o trajeto vetorial da agulha, que pode ser acionado pelo professor durante o treinamento, para posterior análise automatizada. Em versões futuras essa análise poderá ser realizada em tempo real. Uma vez tendo-se o registro do procedimento ideal, realizado por um especialista, pode-se compará-lo à trajetória desenvolvida pelo treinando e obter uma nota, bem como eventuais críticas, do que foi exercitado.

A partir de informações colhidas com especialistas, sabe-se que os dois principais aspectos a avaliar no processo de aproximação e posicionamento da agulha são: proximidade da agulha do ponto alvo na boca e ângulo sólido de posicionamento da agulha (Figura 5). Para se chegar a uma nota entre 0 e 1 são multiplicados três termos. O primeiro recebe um valor 0 ou 1 (0: erro grave, como por exemplo atingir acidentalmente alguma parte do rosto do aluno ou não chegar ao ponto de introdução da agulha). O segundo termo se refere à inclinação do posicionamento da agulha em relação à direção considerada de referência. Esse termo depende do cosseno do ângulo formado pela direção da agulha posicionada pelo aluno em relação à direção de referência, elevado a um coeficiente de calibração. Esse coeficiente definirá a velocidade com que a nota se aproximará de zero à medida em que o ângulo do erro aumente. O terceiro termo contribui com o erro de distanciamento do ponto alvo, obtido pelo complemento da divisão, por um coeficiente de calibração linear relativo à distância entre o ponto de referência e o ponto atingido pelo aluno.

A partir do exposto chegou-se à formulação, apresentada no quadro da Figura 6, para a nota do procedimento.
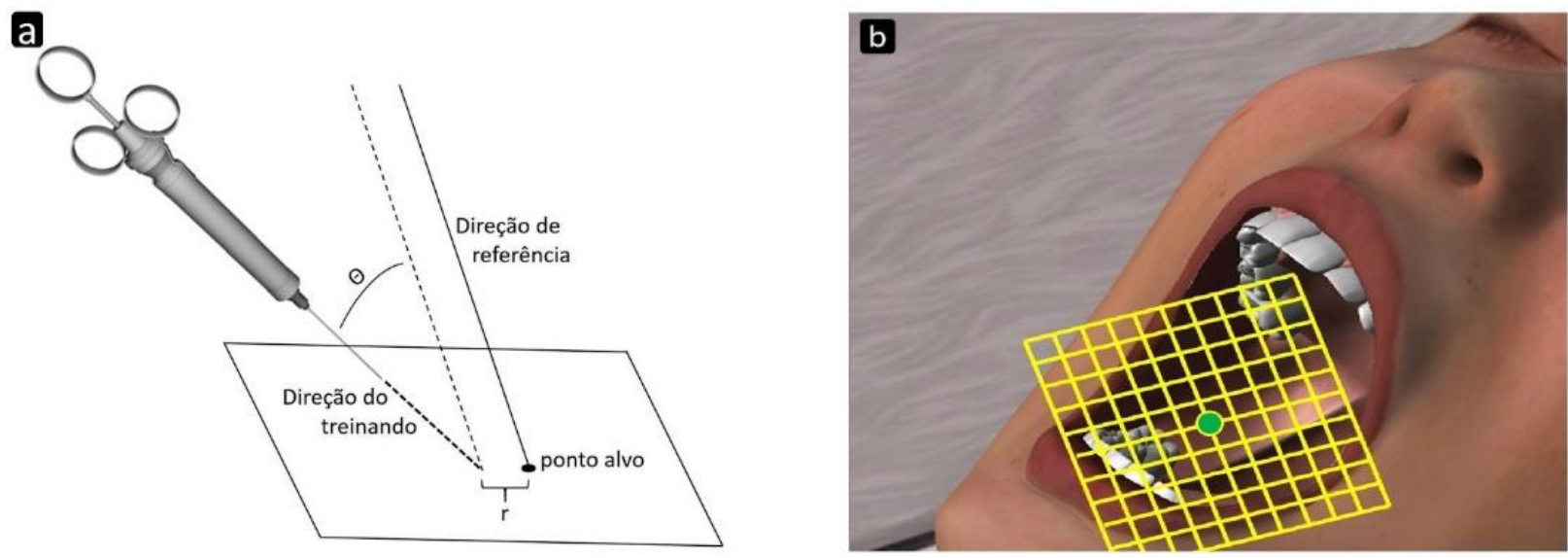

Figura 5: Parâmetros usados na avaliação.. 


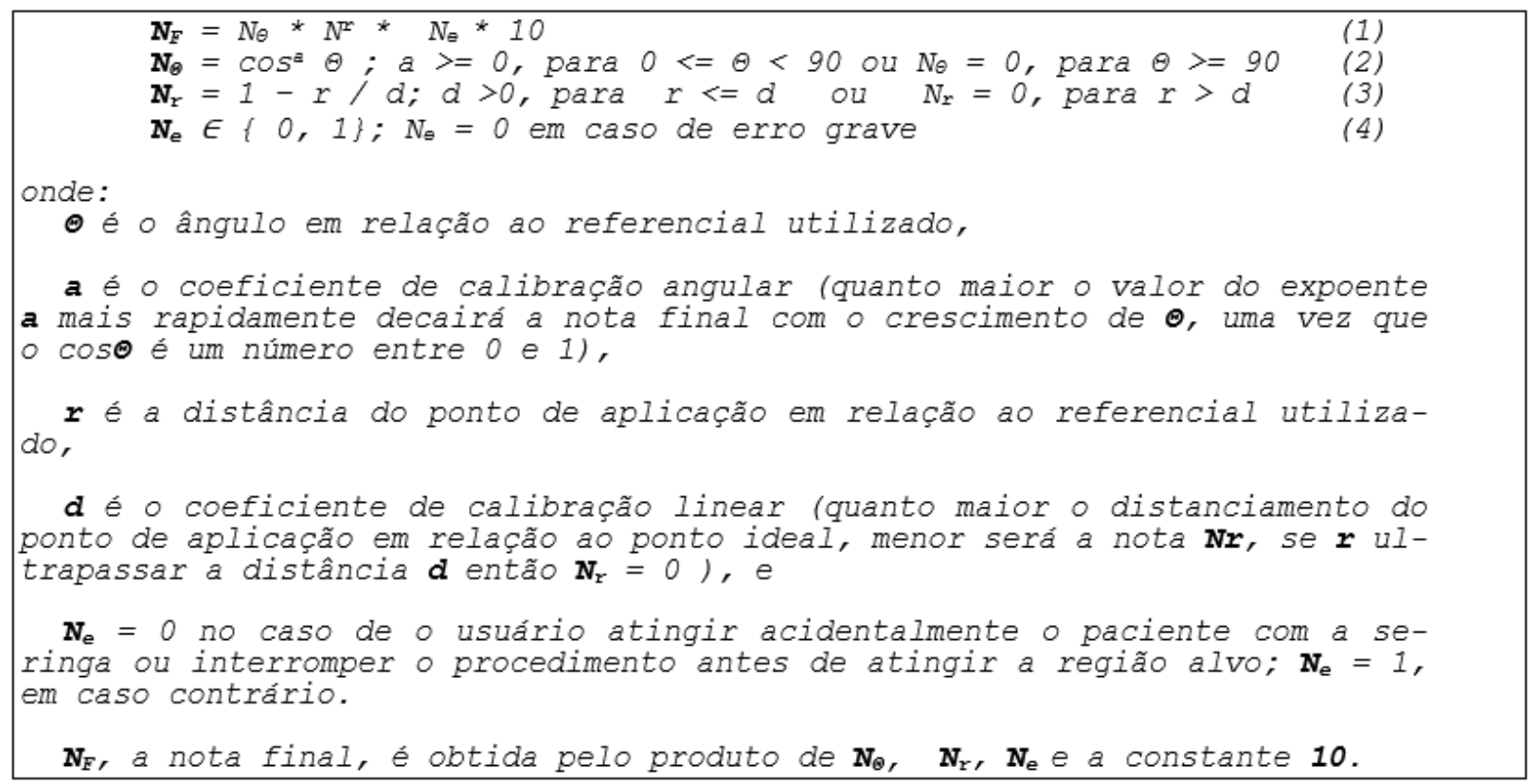

Figura 6: Fórmulas da Avaliação Automática.

Cada uma das componentes da fórmula (Figura 6) pode ser zerada devido a erros graves. $N_{\Theta}$ varia com o cosseno do ângulo formado entre a direção da trajetória da agulha e a direção ideal (definida por especialistas). O coeficiente a permite aumentar o rigor da nota. Se a tender a infinito teremos $N_{\Theta}=1$ apenas no caso de $\Theta=0$, e $N_{\Theta}$ tenderá a zero nos demais ângulos, uma vez que um número entre 0 e 1 , exclusive, elevado a infinito tende a zero. $N_{r}$ avalia a proximidade do ponto da boca tocado pela agulha (no plano exibido na Figura 6b) em relação ao ponto ideal (definido por especialistas). A calibragem de $N_{r}$ se dá pela definição da tolerância $\mathbf{d}$ que indica o raio a partir do ponto ideal dentro do qual ainda será aceitável como ponto de entrada da agulha. Por fim, $N_{e}$ é uma variável discreta, que possui o valor neutro 1 em situações normais, mas assume o valor $\mathbf{0}$ em casos de erros graves ou interrupção do procedimento.

\subsection{Design e Modelagem 3D}

O principal propósito do design neste experimento foi pesquisar, modelar e texturizar uma pequena clínica odonto pediátrica com intuito de enriquecer a imersão do aluno durante seu treinamento de anestesia. Esta parte do projeto foi complementar à pesquisa, feita na FOB-USP, que teve como foco o desenvolvimento do modelo 3D da cabeça de uma criança (A. Tori et al., 2014).

Para essa clínica (Figura 1a) foi preciso desenvolver um ambiente fechado com elementos, em 3D e com poucos polígonos, semelhantes aos encontrados em uma clínica real, tais como uma cadeira odontológica completa, janela, paredes, piso, elementos decorativos e pias. Aliado ao processo de criação da clínica, um corpo infantil simplificado também foi desenvolvido para permitir que o modelo da cabeça fizesse parte do contexto 
da clínica simulada.

A modelagem da cabeça (Figura 1b) é composta pela estrutura óssea do crânio e das camadas de tecidos da face, presentes na região do nervo alveolar inferior, equivalente a apresentada pelo público infantil, na faixa etária dos 07 aos 12 anos, considerando as características físicas e reais apresentadas por cada tecido (tecidos moles, músculos, nervos). A importância dessa modelagem é simular de forma realística a anatomia da face, com a visualização das camadas de tecidos presentes na região citada, proporcionando um ambiente virtual rico para imersão do aluno, durante a realização da técnica de bloqueio dessa região da boca acessado via computador pelo aluno de graduação em odontologia para seu treinamento.

O princípio da metodologia aplicada na modelagem 3D da cabeça foi o levantamento de modelos anatômicos das estruturas que seriam modeladas, desde aulas e livros de anatomia, imagens da internet e principalmente ressonâncias magnéticas. Em seguida, o crânio em 3D, que foi o ponto de partida do processo de modelagem 3D no software Autodesk Maya 2013, foi desenvolvido com o auxílio de esboços em papel. Com o crânio e arcada dentária em 3D finalizados, passou-se para a modelagem dos músculos, com foco na região da boca. As modelagens das veias, artérias e nervos, foram feitas em seguida, permitindo finalizar o processo com as camadas da pele e gengiva. Para o desenvolvimento dos elementos da clínica, diversas imagens de cada um dos objetos separados foram selecionados, incluindo o corpo da criança, e retirados de repositórios disponíveis gratuitamente na internet, já que não houve a necessidade de se modelar muitos detalhamentos destes mesmos objetos. Todas as modelagens foram feitas com um número reduzido de polígonos, para não sobrecarregar o sistema.

Após as modelagens da cabeça construídas e finalizadas, os estudos concentraramse no desenvolvimento das texturas superficiais dos tecidos. As texturas são imagens em formato JPEG, e foram desenvolvidas no software Adobe Photoshop CS6. Para se criar a textura da parte óssea, foi necessário tirar fotos de um crânio real em 5 posições diferentes, para que depois pudesse ser feito uma montagem com todas elas, gerando uma única imagem ou textura. Para o resto da modelagem, incluindo a clínica e o corpo da criança, foi usada a técnica de pintura digital e montagens de imagens de uso livre e permitidas encontradas na internet.

\subsection{Prova de Conceito}

A prova de conceito foi desenvolvida utilizando a plataforma Unity 4.6, na qual foram acrescentadas as bibliotecas do Oculus Rift DK2 e Leap Motion.

O Leap Motion rastreia a mão e dedos do treinando, que deve estar segurando uma seringa carpule real ou, na falta de uma, pode segurar qualquer objeto. Em sincronia com os movimentos do aluno, e na mesma posição do espaço real em torno do mesmo, uma mão virtual reproduz os movimentos de sua mão e dedos, conferindo maior realismo e imersão ao experimento. Acoplada à agulha da ferramenta virtual, há um detector de colisões que identifica a região da cabeça na qual a seringa realiza contato. Os movimentos da seringa foram limitados para que seu corpo não pudesse atravessar nenhum tecido, 
desta forma não interferindo na sensação de imersão.

Sendo o objetivo da simulação a aplicação de anestesia no nervo alveolar inferior, foi implementado no ponto de injeção um plano que, por meio de um script, calcula o ângulo de inclinação da seringa durante a aplicação (Figura 5b), para extração dos parâmetros utilizados pelo módulo de avaliação automática (Figura 5a). Com as informações do rastreamento do Leap Motion, obtém-se também a posição no espaço virtual tridimensional da agulha da seringa em função do tempo.

O programa exporta no final de cada simulação um arquivo texto com as informações previamente detalhadas, utilizando a persistência de dados da linguagem $C$, com a qual foram escritos todos os códigos da simulação. Por fim, foram implementados comandos para escolher qual o tipo de visão desejada: por HMD ou pelo monitor. Para efeito de prova de conceito foram utilizados, para a avaliação automática do procedimento, os coeficientes $\mathbf{a}=2$ e $\mathbf{d}=4$ (a distância $\mathbf{r}$ do ponto de colocação da agulha em relação ao alvo de referência é medida em centímetros).

\section{Experimento}

Para esse experimento foi utilizada uma versão do protótipo em que o Leap Motion estava configurado no modo de detecção de instrumento. Nesse modo apenas a seringa virtual aparecia para o participante, que não via sua mão. Em vez de segurar a seringa carpule os participantes utilizaram um objeto cilíndrico (canudinho de refrigerante) (Figura 1c), que era detectado pelo Leap Motion e substituído pela seringa virtual. Posteriormente ao experimento o módulo de ambiente virtual foi aprimorado, passando a mostrar a mão conforme descrito na seção 5.4 e mostrado na Figura 4.

O objetivo desse experimento foi demonstrar a viabilidade e a aplicabilidade do VIDA Odonto como ferramenta didática, bem como identificar eventuais problemas de interface, interação e usabilidade. A partir desses resultados passaremos ao desenvolvimento da ferramenta completa e robusta a ser implantada no laboratório LaSIT da FOB/USP. Para tanto foi realizada uma pesquisa qualitativa com especialistas (professores e pesquisadores da odontologia) e representantes do público-alvo. Para esse tipo de estudo, conforme conhecido estudo de Nielsen (Nielsen, 2012) não são necessários mais do que 5 participantes.

Primeiramente calibramos o sistema com a trajetória desenvolvida pela professora especialista no procedimento de anestesia, participante da pesquisa e coautora deste artigo. Em seguida os demais especialistas realizaram o procedimento, como se fossem alunos, para que o sistema de avaliação pudesse ser testado, bem como a usabilidade do sistema. Por fim realizamos a pesquisa qualitativa com os alunos.

Inicialmente foi apresentada uma breve explicação sobre o experimento e regras básicas dos testes. Em seguida, os participantes foram orientados a preencher o Termo de Consentimento Livre e Esclarecido. O experimento contou com o auxílio de alunos de iniciação científica, mestrandos e doutorandos envolvidos no desenvolvimento do projeto, para o qual deram o suporte necessário, esclarecendo dúvidas e solucionando 
eventuais problemas de uso do sistema pelos participantes.

Após todas as explicações, incluindo o tempo previsto para realização dos testes, foi dada oportunidade para que o candidato declinasse do convite. Na hipótese de se constatar que determinado voluntário viesse apresentar algum desconforto durante a execução do experimento esse seria dispensado e não seria contabilizado como participante. Foi ainda solicitada ao participante autorização para filmagem do procedimento e para divulgação dessas imagens. Se a pessoa não autorizasse a divulgação de sua imagem, ainda assim o experimento poderia ser realizado. Nesse caso, as imagens e vídeos seriam armazenados apenas para registro e consulta restrita aos pesquisadores, sem utilização em publicações.

Após o preenchimento do termo de consentimento de participação no experimento, os estudantes voluntários foram orientados a preencher um questionário online préexperimento contendo dados pessoais, bem como idade, sexo e curso de graduação, que teve como objetivo traçarmos o perfil do estudante. Antes do experimento propriamente dito os participantes passaram por uma sessão de treinamento, durante a qual receberam informações sobre os objetivos da ferramenta, suas funcionalidades e interface. Em seguida puderam usar livremente o sistema e esclarecer dúvidas.

Durante o experimento o participante realizou o procedimento de aplicação de anestesia do nervo alveolar inferior (uma das mais difíceis de aprender e executar) sentado em uma cadeira, como se estivesse em uma clínica odontológica real, e executou o procedimento duas vezes, uma visualizando um dispositivo de realidade virtual (HMD) e outra olhando para a tela do computador. Além disso o participante interagiu com o sistema por meio de um objeto cilíndrico real representado no meio virtual por uma seringa captada por um sensor de reconhecimento de gestos (Leap Motion) que estava acoplado ao dispositivo de realidade virtual. Todos os dispositivos foram previamente desinfetados com álcool gel (e sempre que o equipamento foi passado de um voluntário a outro).

Cada execução do procedimento foi gravada, usando o recurso do próprio sistema, que registra os movimentos e os reproduz com fidelidade, possibilitando que o procedimento seja revisto com total imersão. Durante a coleta de dados os arquivos contendo os registros das trajetórias passaram pelo algoritmo de avaliação da trajetória e receberam notas (ver seção 5.2).

Finalmente, os especialistas e estudantes foram orientados a preencher um formulário online contendo perguntas dissertativas sobre a experiência de interação do aprendiz com o ambiente de treinamento imersivo.

\section{Resultados}

Utilizando como referência a aplicação da injeção por uma profissional da área de odontologia (Figura 1c), foram calibrados os módulos de avaliação automática da trajetória. Seguindo as diretrizes de Nielsen (Nielsen, 2012) para pesquisas qualitativas de usabilidade, 
foram avaliados 14 participantes, sendo 5 deles especialistas e 9 não especialistas (acima do necessário no caso dos não especialistas, que seriam 5 para cada grupo).

O sistema terá a possibilidade de ser utilizado com diferentes interfaces, com vistas a se adequar a diferentes disponibilidades de recursos. Haverá uma versão mobile (com visualização na tela do celular ou com adaptador de RV) e uma para desktop/notebook (com visualização na tela do monitor ou com dispositivo HMD). Nesse experimento, além de se avaliar a funcionalidade e usabilidade do sistema na versão desktop (a mais completa) foram analisadas as aplicações da injeção com e sem o dispositivo HMD. O objetivo foi identificar o impacto da imersão no desempenho. Como a visualização no dispositivo HMD, além de imersiva é estereoscópica (visualização de profundidade), imagina-se que a precisão dos movimentos e, consequentemente, a avaliação das tarefas realizadas, sejam melhores. Com base nesses resultados iniciais serão desenhados experimentos específicos com validação estatística.

As Figuras 7 e 8 apresentam a comparação entre as notas concedidas para os participantes ao realizar o experimento com e sem o dispositivo HMD. É possível observar que houve uma melhora no desempenho da aplicação da injeção com o uso do dispositivo HMD. Com efeito, nota-se que 11 dos participantes obtiveram uma nota maior. Além disso, os participantes, imediatamente após o experimento, preencheram um questionário contendo as seguintes perguntas dissertativas:

- Apresente dificuldades e problemas encontrados;

- Apresente os pontos fortes do sistema;

- Sugestões;

- Faça suas considerações gerais sobre o sistema. 


\section{Nota dos Especialistas}

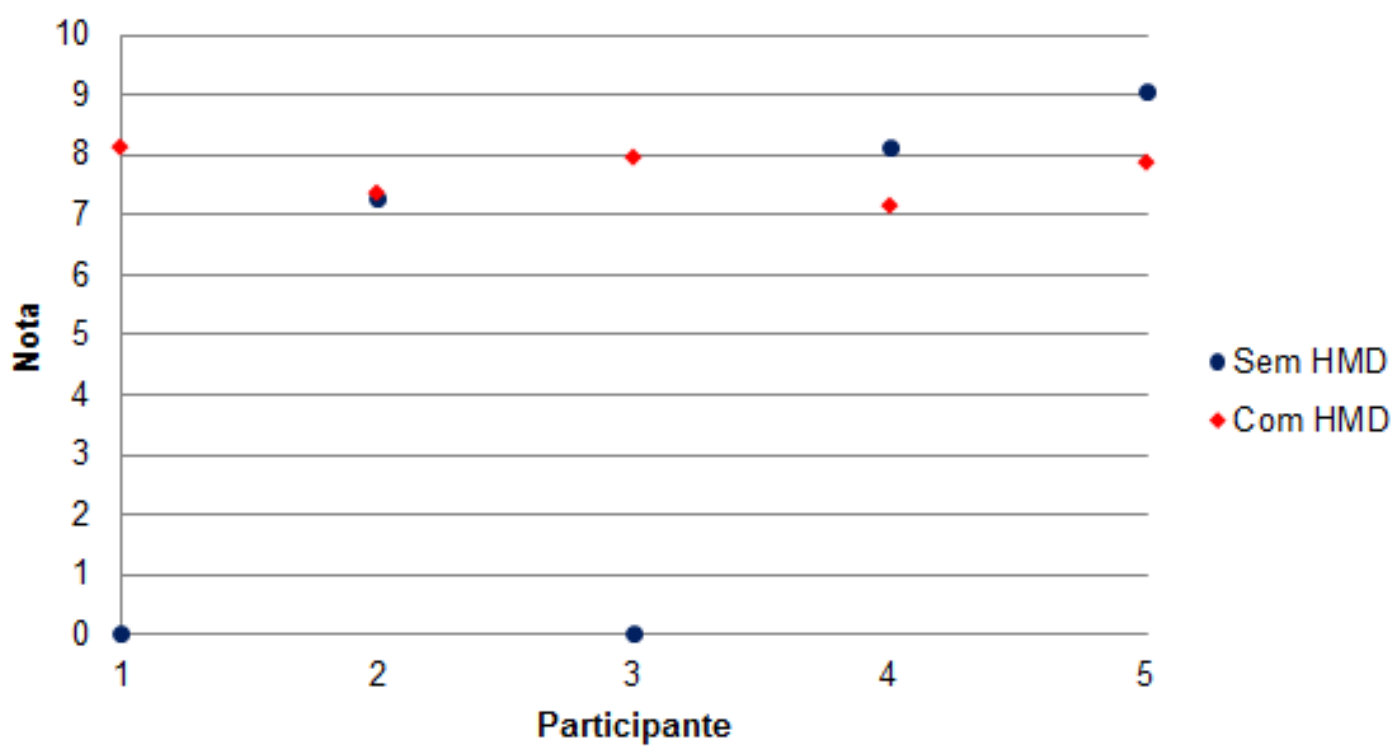

Figura 7: Notas atribuídas aos especialistas que participaram do experimento.

Nota dos Novatos

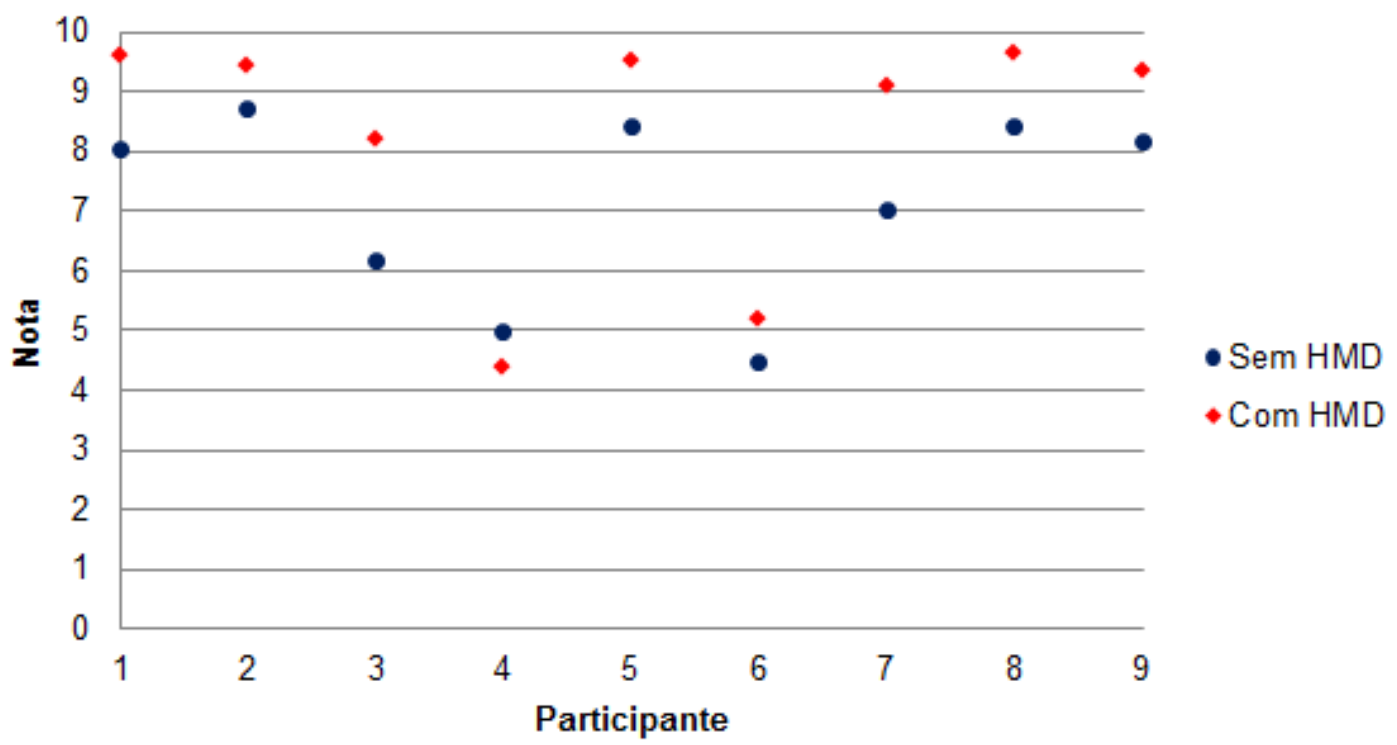

Figura 8: Notas atribuídas aos novatos que participaram do experimento.

A partir de dessas respostas foi identificado que um dos pontos mais levantados pelos participantes foram as dificuldades de se usar o sensor de movimento (Leap Motion) juntamente com o instrumento cilíndrico. Observou-se durante o experimento 
que a seringa virtual apresentava instabilidade por dois motivos: a presença de pontos cegos/ângulos de difícil detecção que tornavam a seringa instável, até quando o participante não tremia, e a leveza do objeto cilíndrico quando comparado à seringa. A utilização do instrumento cilíndrico de forma precisa foi considerada um desafio de difícil adaptação, porém aqueles que já conheciam a tecnologia do sensor demonstraram um domínio e precisão muito maior, indicando que, após um tempo de familiarização, é provável que o participante tenha muito mais facilidade com o equipamento. Na versão atual do VIDA Odonto esse problema já foi eliminado, com o uso de uma seringa carpule real para a realização do treinamento. Apesar disso, entre os participantes mais novos (nascidos em 1996 e 1997) e que não conheciam o sensor, foram feitos comentários positivos quanto à precisão do sensor por três dos seis participantes, sendo estes os únicos comentários positivos sobre o sensor registrados após o experimento. Dois deles, porém, fizeram comentários negativos e o outro se manteve neutro. Ainda assim, esses dados podem demonstrar uma maior aceitação de tecnologias mais novas por alunos jovens (mais próximos da idade alvo deste treinamento) quando comparado a participantes mais velhos.

Outro ponto levantado foi a falta de resistência na perfuração do tecido. Esse tipo de comentário teve origem dos participantes especialistas da área de odontologia que conhecem o projeto Vimet Odonto e já conheciam aparelho de retorno de força háptico, relatando que o retorno de força é bastante relevante para este tipo de experimento. O módulo háptico encontra-se atualmente em desenvolvimento por pesquisadores do Laboratório de Aplicações de Informática em Saúde (LApIS/EACH USP), em colaboração com o Laboratório de Tecnologias Interativas (Interlab/POLI USP), responsável pelo módulo do ambiente virtual e Laboratório de Simulação e Treinamento (LaSIT/FOB USP), onde o sistema será implantado. Também está sendo estudada a inclusão no sistema de uma cabeça de manequim (Ren et al., 2017), com textura e elasticidade de pele similares às de uma pessoas real. Dessa forma o treinando poderá sentir pelo tato a cabeça virtual com a qual estará interagindo. Cabeças de manequim ainda são amplamente utilizadas em treinamento pré-clínico, mas apresentam limitações significativas (Fugill, 2013). Elas não oferecem realimentação clínica realista sobre reações semelhantes às de um paciente real, ou seja, não simulam a dor e desconforto durante um procedimento. Em contraste, a simulação digital interativa pode atender essas necessidades (Fugill, 2013). A ideia é juntar as duas vantagens, o realismo visual e sonoro da RV e o realismo tátil do manequim.

Foram feitos elogios e críticas tanto ao modelo do paciente quanto ao consultório. Em especial, foi observado pelos especialistas que o paciente não apresentava alguns pontos de referência para a aplicação da anestesia. No geral, o ambiente do consultório foi bem aceito e grande parte dos participantes comentou ter a sensação de imersão e presença. Em futuros experimentos essas percepções serão mensuradas com maior precisão. Também foram observados comentários sobre alguns pontos referentes à importância de se ter um simulador para esse tipo de procedimento, a relevância de se criar uma alternativa ao método convencional e a conveniência do auto treinamento que pode ser realizado. 


\section{Conclusão}

Neste trabalho foi apresentado um ambiente de realidade virtual para treinamento denominado VIDA Odonto, o qual mostrou resultados promissores. Os alunos, professores e instituições poderão se beneficiar das abordagens digitais interativas e imersivas, que facilitam a aprendizagem individualizada e a aprendizagem colaborativa, intra e inter instituições, por meio de um software que será disponibilizado em plataforma aberta e gratuita.

O experimento piloto demonstrou a viabilidade de se utilizar tecnologias de realidade virtual para o treinamento de estudantes da área de odontologia na etapa pré-clínica. Observou-se que grande parte dos participantes comentaram ter a sensação de imersão. Esse é um poderoso indicativo para, em um próximo experimento, se analisarem as percepções de "presença"propiciadas pelo sistema, a fim de verificar o quanto o participante falha em perceber a mediação da tecnologia utilizada durante o processo de interação.

Os próximos passos no desenvolvimento do ambiente VIDA Odonto incluem: inclusão de interface háptica, adaptada a uma seringa idêntica à utilizada pelos profissionais durante o procedimento e anestesia; desenvolvimento do módulo do professor, que incluirá o compartilhamento a distância do espaço virtual tridimensional; adaptação para smartphones, usando adaptadores de baixo custo, como o Google Cardboard; estudos com novos dispositivos, como o Hololens da Microsoft; inclusão de um boneco palpável, devidamente registrado com o paciente virtual, possibilitando maior realismo ao treinando.

\section{Agradecimentos}

Os autores agradecem ao CNPq pelas bolsas de produtividade de Romero Tori (processo 311991/2015-7) e de Maria Aparecida A. M. Machado (processo 311251/2016-1), assim como pelas bolsas de iniciação científica de Allan A. Tori (processo 156656/2012-4) e de Gustavo Ziyu Wang (processo 158619/2015-3).

Ao Centro de Estudos Sociedade e Tecnologia (CEST) da Escola Politécnica da USP pela bolsa de Iniciação Científica concedida ao Projeto VIDA Odonto.

Ao Laboratório de Aplicações de Informática em Saúde (LApIS) da EACH/USP e seus pesquisadores, em especial à Profa Fatima Nunes e ao pós-doutorando Cleber G. Corrêa, que participaram do projeto Vimet Odonto e recentemente se integraram ao desenvolvimento do VIDA Odonto, logo após a realização desse primeiro experimento aqui relatado.

Aos professores, pesquisadores e alunos da Faculdade de Odontologia da USP em Bauru, que vem contribuindo com consultoria técnica e avaliação do sistema.

Aos colegas do INTERLAB (Laboratório de Pesquisa em Tecnologias Interativas), em especial ao Prof. Ricardo Nakamura e à funcionária Mariza Ushijima Leone. 
Nosso reconhecimento ao designer Kim Tanabe de Moura Leite, que trabalhou juntamente com um dos autores deste trabalho, Allan Amaral Tori, na modelagem da cabeça do paciente, e a suas co-orientadoras na Iniciação Científica, Professoras Sileide Paccola e Suzy Ribeiro Amantini.

Agradecimentos aos alunos de graduação da Escola Politécnica da USP que contribuíram e vem contribuindo com o desenvolvimento do VIDA Odonto, entre eles: Renan de Araujo Alves, Leonardo Akira Fattore Hirano e Felipe Massao Kitanaka Matsuoka.

\section{References}

Azer, S. A., \& Eizenberg, N. (2007). Do we need dissection in an integrated problem-based learning medical course? perceptions of first-and second-year students. Surgical and Radiologic Anatomy, 29(2), 173-180. doi: 10.1007/s00276-007-0180-x [GS Search]

Bakr, M. M., Massey, W., \& Alexander, H. (2013). Evaluation of simodont $®$ haptic 3d virtual reality dental training simulator. International journal of dental clinics, 5(4). [GS Search]

Battulga, B., Konishi, T., Tamura, Y., \& Moriguchi, H. (2012). The effectiveness of an interactive 3-dimensional computer graphics model for medical education. Interactive journal of medical research, 1(2). doi: 10.2196/ijmr.2172 [GS Search]

Biocca, F., Harms, C., \& Gregg, J. (2001). The networked minds measure of social presence: Pilot test of the factor structure and concurrent validity. In 4th annual international workshop on presence, philadelphia, pa (pp. 1-9). [GS Search]

Bowman, D. A., Kruijff, E., LaViola Jr, J. J., \& Poupyrev, I. (2001). An introduction to 3-d user interface design. Presence: Teleoperators and virtual environments, 10(1), 96-108. doi: 10.1162/105474601750182342 [GS Search]

Correa, C. G. (2015). Simulação de inserção de agulha para treinamento de procedimento de anestesia odontológica. Universidade de São Paulo.

Finkelstein, P., \& Mathers, L. H. (1990). Post-traumatic stress among medical students in the anatomy dissection laboratory. Clinical Anatomy, 3(3), 219-226. doi: 10.1002/ca.980030308 [GS Search]

Fugill, M. (2013). Defining the purpose of phantom head. European Journal of Dental Education, 17(1). doi: 10.1111/eje.12008 [GS Search]

Gustavson, N. (1988). The effect of human dissection on first-year students and implications for the doctor-patient relationship. Academic Medicine, 63(1), 62-4. doi: 10.1097/00001888-198801000-00011 [GS Search]

Hancock, D., Williams, M., \& Taylor, A. (1998). Psychological impact of cadavers and prosections on physiotherapy and occupational therapy students. Australian Journal of Physiotherapy, 44(4), 247-255. doi: 10.1016/s0004-9514(14)60384-6 [GS Search]

McLachlan, J. C., Bligh, J., Bradley, P., \& Searle, J. (2004). Teaching anatomy without cadavers. Medical education, 38(4), 418-424. doi: 10.1046/j.1365-2923.2004.01795.x [GS Search]

Melo, C., Aceiro, J., Nunes, F., Tori, R., \& Muntoreanu, I. (2011). Implementação de atlas virtual: Definição de procedimentos e ações para o ensino da assistência ao parto. 
VII Congresso Brasileiro de Enfermagem Obstétrica e Neonatal, 4291-4297. [GS Search] Mirghani, I., Mushtaq, F., Allsop, M., Al-Saud, L., Tickhill, N., Potter, C., ... Manogue, M. (2016). Capturing differences in dental training using a virtual reality simulator. European Journal of Dental Education. doi: 10.1111/eje.12245 [GS Search]

Nielsen, J. (2012). How many test users in a usability study. Nielsen Norman Group, 4(06). [GS Search]

Pereira, L. A. P. (2016). Criação, desenvolvimento, aplicação e validação de um simulador computadorizado de realidade virtual para o ensino e treinamento de bloqueio do nervo alveolar inferior. Universidade Estadual de Campinas.

Poyade, M., Lysakowski, A., \& Anderson, P. (2014). Development of a haptic training simulation for the administration of dental anaesthesia based upon accurate anatomical data. In Proceedings of the eurovr - conference and exhibition of the european association of virtual and augmented reality (p. 143-7). [GS Search]

Rebelo, I. B., \& Pinho, M. S. (2004). Interação em ambientes virtuais imersivos. Realidade Virtual-Conceitos e Tendências. Editora Mania de Livro: São Paulo. [GS Search]

Ren, Q., Wang, Y., Zheng, Q., Ye, L., Zhou, X., \& Zhang, L. (2017). Survey of student attitudes towards digital simulation technologies at a dental school in china. European Journal of Dental Education, 21(3), 180-186. doi: 10.1111/eje.12198 [GS Search]

Tori, A., Leite, K., Pacolla, S., Amantini, S. R., \& Machado, M. A. (2014). Modelagem realística de cabeça infantil para aplicação em simulador de anestesia odontológica. Iniciação-Revista de Iniciação Científica, Tecnológica e Artística, 4(3). [GS Search]

Tori, R. (2010). Educação sem distância: as tecnologias interativas na redução de distâncias em ensino e aprendizagem. SENAC. [GS Search]

Tori, R., Marques, F., Nakamura, R., Bernardes, J., Correa, C., \& Tokunaga, D. (2009). Design de interaçao para um atlas virtual de anatomia usando realidade aumentada e gestos. Interaction South America, 9, 1-12. [GS Search]

Tori, R., Nunes, F. L., Gomes, V. H., \& Tokunaga, D. M. (2009). Vida: Atlas anatômico 3d interativo para treinamento a distância. In Anais do workshop de informática na escola (Vol. 1, pp. 1801-1810). [GS Search]

Tori, R., Wang, G., Sallaberry, L., Tori, A., de Oliveira, E. C., \& Machado, M. A. d. A. M. (2016). Treinamento odontológico imersivo por meio de realidade virtual. In Brazilian symposium on computers in education (simpósio brasileiro de informática na educação-sbie) (Vol. 27). doi: 10.5753/cbie.sbie.2016.400 [GS Search]

Vora, J., Nair, S., Gramopadhye, A. K., Duchowski, A. T., Melloy, B. J., \& Kanki, B. (2002). Using virtual reality technology for aircraft visual inspection training: presence and comparison studies. Applied ergonomics, 33(6), 559-570. doi: 10.1016/s00036870(02)00039-x [GS Search]

$\mathrm{Xu}$, D. (2006). A neural network approach for hand gesture recognition in virtual reality driving training system of spg. In Pattern recognition, 2006. icpr 2006. 18th international conference on (Vol. 3, pp. 519-522). doi: 10.1109/icpr.2006.109 [GS Search] 